\title{
All-Arthroscopic Treatment of Intra- and Extra- Articular Localized Villonodular Synovitis of Knee
}

\author{
Roberto Simonetta ${ }^{1}$ Michela Florio ${ }^{2}$ Filippo Familiari ${ }^{3}$ Giorgio Gasparini ${ }^{3}$ Michele Attilio Rosa ${ }^{2}$ \\ ${ }^{1}$ Department of Orthopaedic and Trauma Surgery, C.O.T. Cure \\ Ortopediche Traumatologiche, Messina, Italy \\ 2 Department of Orthopaedic \& Trauma Surgery, Messina University, \\ Messina, Italy

\begin{abstract}
Address for correspondence Filippo Familiari, MD, Department of Orthopaedic and Trauma Surgery, Magna Graecia University of Catanzaro, Campus "S. Venuta," Viale Europa (Loc.Germaneto), 88100 Catanzaro, Italy (e-mail: filippofamiliari@gmail.com).
\end{abstract}

${ }^{3}$ Department of Orthopaedic and Trauma Surgery, Magna Graecia University of Catanzaro, Catanzaro, Italy

Joints 2017;5:184-187.

\begin{abstract}
Keywords

- pigmented villonodular synovitis

- intra-articular

- extra-articular

- arthroscopic treatment

Pigmented villonodular synovitis (PVNS) is a rare, benign, proliferative neoplastic condition affecting synovial-lined anatomic spaces. PVNS is characterized by hypertrophy of a synovial membrane by villous, nodular, and villonodular proliferation, with pigmentation secondary to hemosiderin deposition. The two forms of PVNS that have been described are diffuse (DPVNS) and localized (LPVNS). The knee is the most commonly involved anatomic location, followed by hip, ankle, shoulder, and elbow. Diagnosis of PVNS is not always obvious clinically. Various imaging modalities are often necessary to exclude other conditions and narrow the diagnosis. Magnetic resonance imaging has become the modality of choice for diagnosing PVNS. We present a case of intra-articular LPVNS with an extra-articular extension through the posterior capsule that has been successfully removed in an all-arthroscopic fashion.
\end{abstract}

\section{Introduction}

Pigmented villonodular synovitis (PVNS) is a rare, benign, proliferative neoplastic condition affecting synovial-lined anatomic spaces. ${ }^{1,2}$ It may cause swelling, pain, and stiffness. PVNS is characterized by hypertrophy of the synovial membrane by villous, nodular, and villonodular proliferation, with pigmentation secondary to hemosiderin deposition. The annual incidence of PVNS in the United States is 1.8 to 2.0 per $1,000,000$ people. ${ }^{3,4}$

The two forms of PVNS that have been described are diffuse (DPVNS) and localized (LPVNS). PVNS is also categorized as intra-articular and extra-articular. It is usually monoarticular, affecting adults in the third or fourth decade of life; the knee is the most commonly involved anatomic location, followed by hip, ankle, shoulder, and elbow. 5,6

Histologically, LPVNS and DPVNS are similar; however, they differ in their clinical presentation, prognosis, and response to treatment. In the knee, LPVNS lesions occur most commonly in the region of the anterior horn of the medial meniscus. LPVNS usually presents with symptoms of pain and swelling, but it more commonly presents with locking, catching, and instability. ${ }^{7-15}$ DPVNS is characterized by involvement of most or all of the joint synovium. Swelling and pain are more pronounced than in LPVNS and usually are poorly localized. DPVNS tends to have a more rapidly destructive course, and as a result, has a poorer prognosis. DPVNS can also present with extra-articular extension, either at the time of primary diagnosis or as recurrent disease. Extra-articular DPVNS can encroach on major neurovascular structures, making surgical excision more challenging and complete excision difficult. ${ }^{16}$

PVNS is very difficult to diagnose and correctly manage. MRI should always be performed in the case of a suspicion of PVNS. It is particularly helpful in distinguishing between DPVNS and LPVNS as well as in determining the presence of extra-articular involvement.

The goals of treatment of PVNS are to reduce pain, stiffness, and arthritic progression and increase functional outcomes. Several treatment options are currently available; the appropriate treatment of each patient must be based on the type of PVNS (i.e., DPVNS or LPVNS), presence of extra- published online August 8, 2017
DOI https://doi.org/ $10.1055 / \mathrm{s}-0037-1605586$ ISSN $2282-4324$
Copyright @ 2017 Georg Thieme Verlag KG Stuttgart · New York
License terms

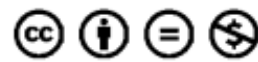


articular extensions of the disease, and the level of experience of the surgeon.

We present a case of intra-articular LPVNS with an extraarticular extension through the posterior capsule that has been successfully removed in an all-arthroscopic fashion.

\section{Case Report}

We present a case of intra-articular LPVNS with an extraarticular extension through the posterior capsule in a 36-yearold woman who had been complaining of moderate pain on her left knee for 2 years. The patient was informed that data concerning the case would be submitted for publication and she provided the consent.The patient recalled no recent injury or antecedent trauma and her medical history was unremarkable. Physical examination revealed moderate, recurrent swelling of the left knee without surrounding warmth. The patient had decreased range of motion (ROM) in flexion (0-90 degrees on the left knee compared with 0-120 degrees on the right knee). Radiographic findings of the left knee were normal, without a visible soft-tissue mass, bone erosion, acute fracture, dislocation, or effusion. An MRI study of the left knee revealed slight and diffused joint effusion with nonspecific synovial proliferation and distension of the popliteal fossa due to the presence of an egg-shaped mass $(7.82 \times 2.97 \mathrm{~cm})$. The mass demonstrated low-signal intensity on both T1-weighted and T2-weighted fat-saturated images ( - Fig. 1 ).

The patient was taken to the operating room for an arthroscopic biopsy of the mass. The biopsy was performed using two portals (anterosuperior and anteroinferior). Both portals were established on the medial side of the knee to facilitate the skin incision, eventually needed for an open excision of the mass. The anterior intra-articular structures appeared normal on arthroscopic examination. An irregular, tan-yellow, rubbery soft tissue mass was located behind the cruciate ligaments. Biopsy samples were collected and sub- mitted for histopathologic examination that showed cells with nuclei with dispersed chromatin and evident cytoplasms, abundant macrophages loaded with hemosiderin, and giant cells of variable size.

On the basis of histopathologic findings, a diagnosis of PVNS was favored, and operative excision was recommended. The patient was taken a second time to the operating room for excision of the mass. The patient was placed in the supine position with no leg holder to allow maximum flexion of the knee. The procedure was performed through an all-arthroscopic fashion using four arthroscopic portals (anteromedial, anterolateral, posteromedial, and posterolateral), switching them as viewing and working portals when needed. During the procedure, the mass showed to extension from intra- to extra-articular through a single hole in the posterior capsule. The mass was excised completely in an allarthroscopic fashion, and the extra-articular portion was removed through the hole in the posterior capsule. Histologic examination was performed on surgical samples and diagnosis of PVNS was confirmed.

Immediately after surgery, patient showed full ROM of the knee. At the 24-month follow-up, patient had no complications, with a pain-free and full return of knee and lower extremity function and no clinical or radiologic evidence of recurrence (-Fig. 2 ).

\section{Discussion}

Diagnosis of PVNS is not always obvious. Patients usually present with some or all of the following symptoms: pain (often worse at night), swelling, locking, catching, instability, decreased ROM, joint line tenderness, and/or a palpable mass. ${ }^{17}$ Notably, patients may commonly tolerate several years of symptoms before seeking medical attention. ${ }^{18}$ Notably, Schwartz et al reported a 5-year mean duration of symptoms before the patient presented for treatment. ${ }^{19}$

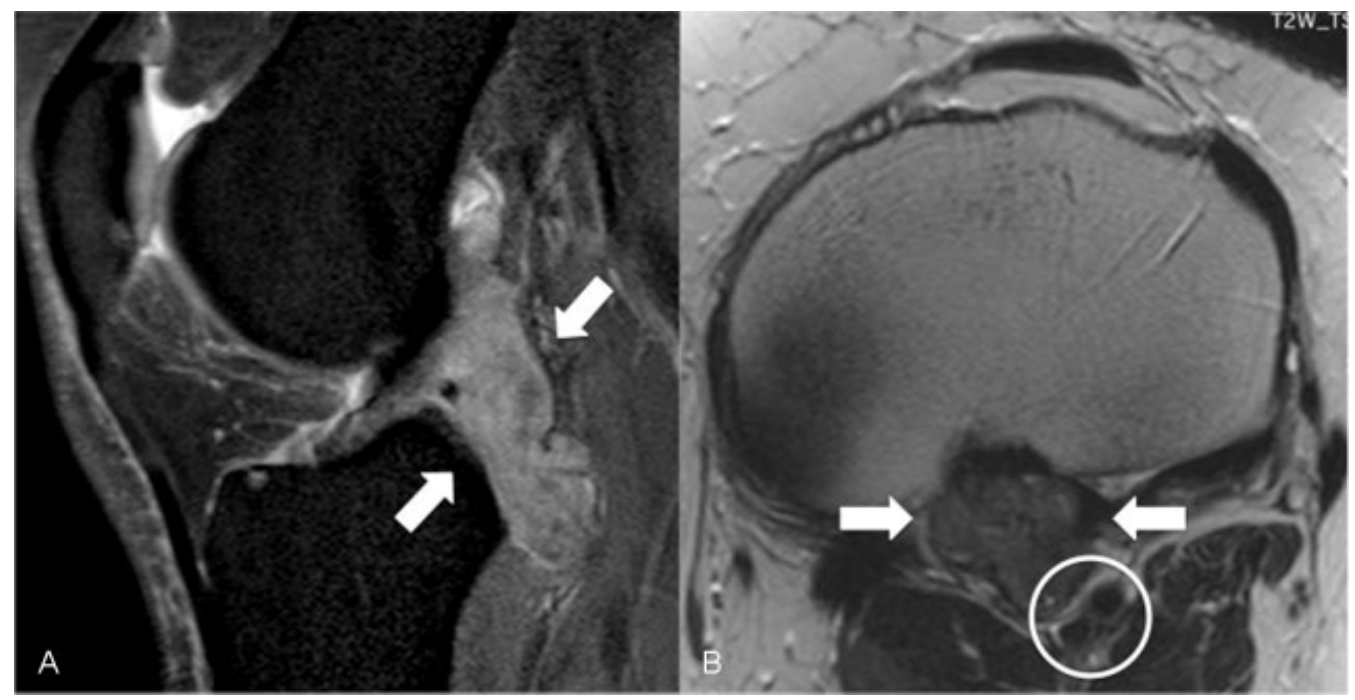

Fig. 1 (A) A sagittal T2-weighted fat-saturated magnetic resonance image of the left knee showing a lobulated, enhancing mass (white arrows) in the posterior aspect of the knee. (B) An axial T1-weighted magnetic resonance image of the left knee showing the neurovascular bundle (circle) displaced by the mass (arrows). 

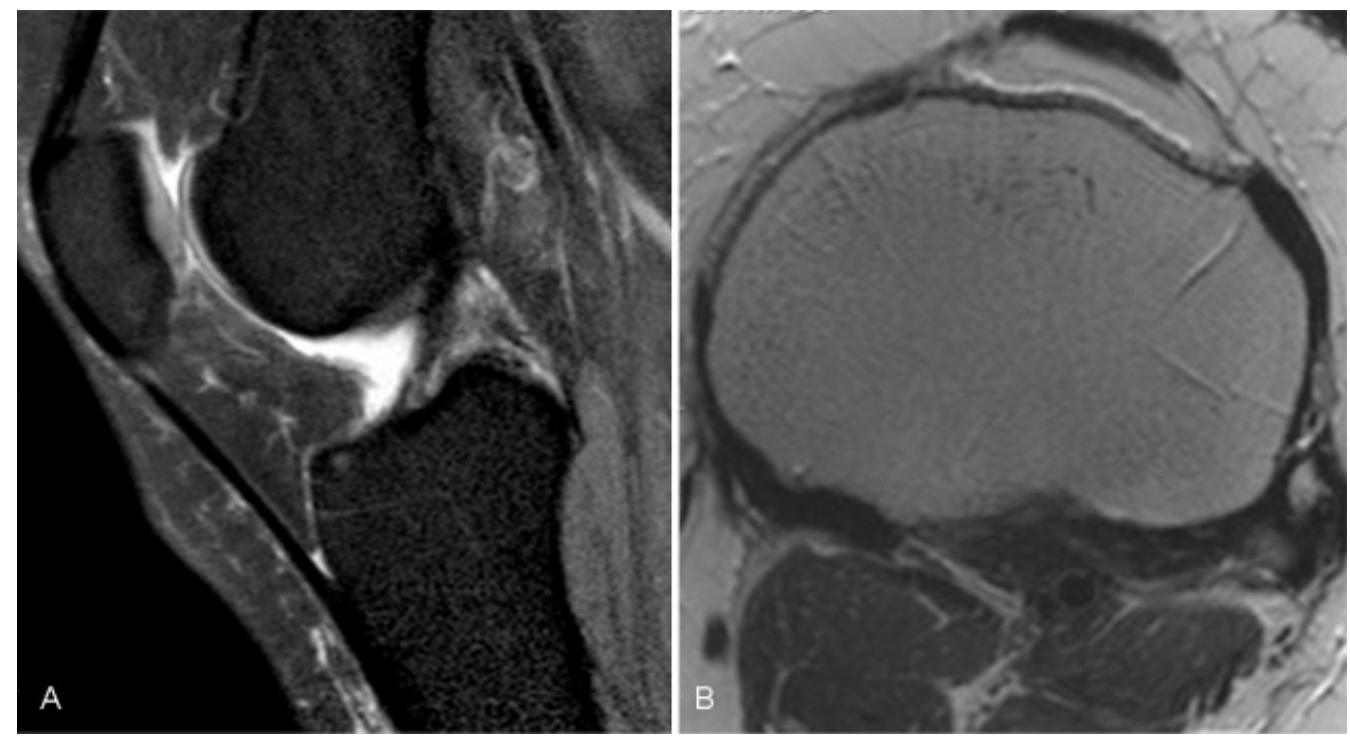

Fig. 2 (A) Postoperative sagittal T2-weighted fat-saturated and (B) axial T1-weighted magnetic resonance images of the left knee showing complete removal of intra- and extra-articular PVNS from the left knee 18 months after an all-arthroscopic procedure. The patient was working full time with no symptoms. PVNS, pigmented villonodular synovitis.

Various imaging modalities are often necessary to exclude other conditions (e.g., loose body, osteochondral fragment, displaced meniscal tear, and soft tissue mass) and narrow the diagnosis. The majority of cases have no plain radiographic findings, ${ }^{20}$ and magnetic resonance imaging (MRI) has become the modality of choice for diagnosing PVNS: "Blooming" or accentuation of signal reduction on gradient echo sequences due to magnetic susceptibility artifact from hemosiderin is typical of PVNS. ${ }^{21}$ MRI can also be helpful in determining the extent of disease and distinguishing DPVNS from LPVNS. Typical MRI findings for LPVNS include a periarticular or synovial nodular mass with varying degrees of bone erosion. The high hemosiderin content causes the mass to appear as either a spotty or an extensive low signal on T1- and T2-weighted images in both DPVNS and LPVNS. ${ }^{21}$ Classically, it is described as "dark on dark" on T1- and T2-weighted images.

The goal behind the management of PVNS is to eradicate all abnormal synovial tissue to relieve pain, lower the risk of joint destruction, and avoid local recurrence. Although surgical synovectomy has been proven effective, the type of resection required remains controversial. Anterior and posterior open arthrotomy and synovectomy under direct visualization have been performed with recurrence rates similar to the best reported for this condition. ${ }^{22-24}$ Open techniques are especially important in the presence of extraarticular extension of disease, which cannot be easily accessed with an arthroscope. ${ }^{22,25}$ Other authors have reported similar recurrence rates with all-arthroscopic total synovectomy. ${ }^{25-27}$ Still others have reported similar recurrence rates combining the two techniques using a posterior open and anterior arthroscopic approach. ${ }^{24}$

As shown in the present case, the all-arthroscopic approach can be very effective in diagnosing and treating LPVNS of the knee.

\section{References}

1 Murphey MD, Rhee JH, Lewis RB, Fanburg-Smith JC, Flemming DJ, Walker EA. Pigmented villonodular synovitis: radiologic-pathologic correlation. Radiographics 2008;28(05):1493-1518

2 O'Connell JX. Pathology of the synovium. Am J Clin Pathol 2000; 114(05):773-784

3 Lin J, Jacobson JA, Jamadar DA, Ellis JH. Pigmented villonodular synovitis and related lesions: the spectrum of imaging findings. Am J Roentgenol 1999;172(01):191-197

4 Bravo SM, Winalski CS, Weissman BN. Pigmented villonodular synovitis. RadiolClin North Am 1996;34(02):311-326, x-xi

5 Rydholm U. Pigmented villonodular synovitis. Acta Orthop Scand 1998;69(02):203-210

6 Abdelwahab IF, Kenan S, Steiner GC, Abdul-Quader M. True bursal pigmented villonodular synovitis. Skeletal Radiol 2002;31 (06):354-358

7 Moskovich R, Parisien JS. Localized pigmented villonodular synovitis of the knee. Arthroscopic treatment. Clin Orthop Relat Res $1991 ;(271): 218-224$

8 Beguin J, Locker B, Vielpeau C, Souquieres G. Pigmented villonodular synovitis of the knee: results from 13 cases. Arthroscopy 1989;5(01):62-64

9 Kim SJ, Shin SJ, Choi NH, Choo ET. Arthroscopic treatment for localized pigmented villonodular synovitis of the knee. Clin Orthop Relat Res 2000;(379):224-230

10 Parikh SN, Chen AL, Ergas E. Localized pigmented villonodular synovitis: arthroscopic diagnosis and management of an "invisible" lesion. Arthroscopy 2002;18(06):E31

11 Delcogliano A, Galli M, Menghi A, Belli P. Localized pigmented villonodular synovitis of the knee: report of two cases of fat pad involvement. Arthroscopy 1998;14(05):527-531

12 Aşik M, Erlap L, Altinel L, Cetik O. Localized pigmented villonodular synovitis of the knee. Arthroscopy 2001;17(06):E23

13 Flandry F, Hughston JC. Pigmented villonodular synovitis. J Bone Joint Surg Am 1987;69(06):942-949

14 Granowitz SP, D'Antonio J, Mankin HL. The pathogenesis and long-term end results of pigmented villonodular synovitis. Clin Orthop Relat Res 1976;(114):335-351

15 Myers BW, Masi AT. Pigmented villonodular synovitis and tenosynovitis: a clinical epidemiologic study of 166 cases and literature review. Medicine (Baltimore) 1980;59(03):223-238 
16 O'Sullivan B, Cummings B, Catton C, et al. Outcome following radiation treatment for high-risk pigmented villonodular synovitis. Int J Radiat Oncol Biol Phys 1995;32(03):777-786

17 Dines JS, DeBerardino TM, Wells JL, et al. Long-term follow-up of surgically treated localized pigmented villonodular synovitis of the knee. Arthroscopy 2007;23(09):930-937

18 Muscolo DL, Makino A, Costa-Paz M, Ayerza MA. Localized pigmented villonodular synovitis of the posterior compartment of the knee: diagnosis with magnetic resonance imaging. Arthroscopy 1995;11(04):482-485

19 Schwartz HS, Unni KK, Pritchard DJ. Pigmented villonodular synovitis. A retrospective review of affected large joints. ClinOrthopRelat Res 1989;(247):243-255

20 Tyler WK, Vidal AF, Williams RJ, Healey JH. Pigmented villonodular synovitis. J Am Acad Orthop Surg 2006;14(06):376-385

21 Cheng XG, You YH, Liu W, Zhao T, Qu H. MRI features of pigmented villonodular synovitis (PVNS). Clin Rheumatol 2004;23(01):31-34

22 Chin KR, Barr SJ, Winalski C, Zurakowski D, Brick GW. Treatment of advanced primary and recurrent diffuse pigmented villonod- ular synovitis of the knee. J Bone Joint Surg Am 2002;84-A(12): 2192-2202

23 Flandry FC, Hughston JC, Jacobson KE, Barrack RL, McCann SB, Kurtz DM. Surgical treatment of diffuse pigmented villonodular synovitis of the knee. Clin Orthop Relat Res 1994;(300): 183-192

24 Sharma V, Cheng EY. Outcomes after excision of pigmented villonodular synovitis of the knee. Clin Orthop Relat Res 2009; 467(11):2852-2858

25 Ogilvie-Harris DJ, McLean J, Zarnett ME. Pigmented villonodular synovitis of the knee.The results of total arthroscopic synovectomy, partial, arthroscopic synovectomy, and arthroscopic local excision. J Bone Joint Surg Am 1992;74(01):119-123

26 De Ponti A, Sansone V, Malcherè M. Result of arthroscopic treatment of pigmented villonodular synovitis of the knee. Arthroscopy 2003;19(06):602-607

27 Zvijac JE, Lau AC, Hechtman KS, Uribe JW, Tjin-A-Tsoi EW. Arthroscopic treatment of pigmented villonodular synovitis of the knee. Arthroscopy 1999;15(06):613-617 